\title{
Development and validation of a nomogram for predicting the risk of obstructive sleep apnea in patients with type 2 diabetes
}

\author{
Huan Shi", Shoukui Xiang", Xiaolin Huang, Long Wang, Fei Hua, Xiaohong Jiang \\ Department of Endocrinology, First People's Hospital of Changzhou, Changzhou, China \\ Contributions: (I) Conception and design: F Hua, X Jiang; (II) Administrative support: F Hua; (III) Provision of study materials or patients: X Jiang; (IV) \\ Collection and assembly of data: H Shi, X Huang; (V) Data analysis and interpretation: H Shi, S Xiang; (VI) Manuscript writing: All authors; (VII) \\ Final approval of manuscript: All authors. \\ "These authors contributed equally to this work. \\ Correspondence to: Fei Hua; Xiaohong Jiang. Department of Endocrinology, First People's Hospital of Changzhou, Changzhou, China. \\ Email: czhuafei@vip.sina.com; 1617141689@qq.com.
}

\begin{abstract}
Background: Obstructive sleep apnea (OSA) is highly prevalent among patients with type 2 diabetes mellitus (T2DM) in China, but few patients with clinical symptoms of OSA are referred for diagnostic polysomnography (PSG). Thus, this study aimed to develop and validate an easy-to-use nomogram that predicts the severity of OSA in patients with T2DM.

Methods: This retrospective study included consecutive patients with T2DM admitted to the Endocrinology Department, Third Affiliated Hospital of Soochow University between January 1, 2016 and December 31, 2019. OSA was diagnosed with PSG. Participants were randomly assigned to a training cohort (70\%) and a validation cohort (30\%). Demographic, anthropometric, and biochemical data were collected. A least absolute shrinkage and selection operator (LASSO) regression model was used to reduce data dimensionality and identify factors for inclusion in the nomogram (training cohort). Nomogram validation was performed in the validation cohort.
\end{abstract}

Results: The study included 280 participants in the training group and 118 participants in the validation group. OSA prevalence was $58.5 \%$. LASSO regression identified waist-to-hip ratio (WHR), smoking status, body mass index (BMI), serum uric acid (UA), the homeostasis model assessment insulin resistance index (HOMA-IR), and history of fatty liver disease as predictive factors for inclusion in the nomogram. Discrimination and calibration in the training group $(\mathrm{C}$-index $=0.88)$ and validation group $(\mathrm{C}$-index $=0.881)$ were good. The nomogram identified patients with T2DM at risk for OSA with an area under the curve of 0.851 [95\% confidence interval (CI), 0.788-0.900].

Conclusions: Our nomogram could be used to facilitate individualized prediction of OSA risk in patients with T2DM and help prioritize patients for diagnostic PSG.

Keywords: Obstructive sleep apnea (OSA); type 2 diabetes mellitus (T2DM); risk; nomogram

Submitted Aug 06, 2020. Accepted for publication Nov 28, 2020.

doi: 10.21037/atm-20-6890

View this article at: http://dx.doi.org/10.21037/atm-20-6890

\section{Introduction}

Obstructive sleep apnea (OSA) is a disorder characterized by upper airway obstruction during sleep that results in breathing pauses, intermittent hypoxia, and fragmented sleep $(1,2)$. The estimated prevalence of OSA in the general population is $9-38 \%$, and the disorder is more common in men, older people, and those with higher body mass index (BMI) (3). Although a lower OSA prevalence of 3-7\% has been reported in China (4), this is likely an underestimate due to people being unaware that they have the disorder or not seeking treatment (5). 
OSA is a sleep and respiratory disorder with high incidence, which can cause damage to multiple system, leading to complications such as hypertension, coronary heart disease, arrhythmia, pulmonary arterial hypertension, stroke, metabolic syndrome and psychological abnormality (6). The pathogenesis was poorly defined. Impairment in upper airway anatomy is the mean cause of OSA. Other causes such as impaired upper airway muscle function and unstable respiratory control play a contributory role (7). Craniofacial shape, obesity, pulmonary function and ventilatory control, genetics and irregular blood glucose control are common risk factors for OSA (8). OSA should be subject to longterm, multidisciplinary treatment management. Obesity is an independent risk factor for OSA, so weight control is of vital importance. other treatments including diet control, strengthening exercise, quit drinking and smoking. Noninvasive positive airway pressure therapy is initial choice for adult OSA patients. An oral appliance that keeps the jaw forward during sleep and a surgery to enlarge the upper airway are other choices. While in the United States, hypoglossal nerve stimulation is an effective treatment for some patients with a body mass index (BMI) less than $32 \mathrm{~kg} / \mathrm{m}^{2}(9)$.

Previous research has indicated that short-term sleep deprivation and fragmentation can induce insulin resistance and that extremes of habitual sleep duration are associated with type 2 diabetes mellitus (T2DM) (10). Furthermore, there is strong evidence that OSA is associated with metabolic syndrome, T2DM, and diabetic renal disease (11-14). It has been proposed that sleep fragmentation and chronic intermittent hypoxia result in the activation of the sympathoadrenal system, elevated oxidative stress, systemic inflammatory responses, and changes in adipokine levels that increase the risk of T2DM (15-17). Notably, the prevalence of OSA among outpatients with T2DM in China was found to be around 60-67\%, and many of these cases had not been previously diagnosed $(18,19)$.

Severe OSA is associated with significantly higher risks of cardiovascular disease, major adverse cardiovascular outcomes, stroke, sudden cardiac death, and all-cause mortality (20), highlighting the importance of the early diagnosis and management of OSA. A definitive diagnosis of OSA relies on polysomnography (PSG) (21); however (22), as this is an expensive and labor-intensive technique that is not widely available, patient access to PSG is limited and many patients with OSA remain undiagnosed. A variety of questionnaires have been developed for use in triage to predict the risk of OSA and thus identify high-risk patients that need referral for PSG, but these approaches are recognized to have limitations $(23,24)$. Different questionnaires focus on different aspects, leading to different sensitivities. Alternative approaches to screening for OSA have included nomograms based on clinical data such as symptom history, demographic characteristics, anthropometric measurements, biochemical investigations, and spirometry data (25-27). obesity is a risk factor for OSA, therefore there was an article using a nomogram to study the incidence of OSA in obese patients (28). However, no previous studies have developed a nomogram tailored for use in patients with T2DM.

The aim of our study was thus to construct and validate an easy-to-use nomogram based on a constellation of objective demographic, biochemical, and anthropometric parameters that could be used to accurately predict the risk of OSA in patients with T2DM. It was anticipated that this nomogram could be used in the clinic to reduce the number of missed OSA diagnoses in patients with T2DM by identifying patients at high risk of OSA who should be referred for PSG. We present the following article in accordance with the TRIPOD reporting checklist (available at http://dx.doi.org/10.21037/atm-20-6890).

\section{Methods}

\section{Study design and participants}

This retrospective study included consecutive patients with T2DM admitted to the Endocrinology Department, Third Affiliated Hospital of Soochow University, Changzhou, China between January 1, 2016 and December 31, 2019. The diagnosis of T2DM was made in accordance with the American Diabetes Association (ADA) criteria (29). The exclusion criteria were the following: (I) prior diagnosis or treatment of OSA, (II) severe congestive heart failure, (III) severe pulmonary disease, (IV) severe chronic kidney disease, (V) severe hepatic disease, and (VI) pregnancy. All procedures performed in this study involving human participants were in accordance with the Declaration of Helsinki (as revised in 2013). The protocol was approved by the Ethics Committee of the Third Affiliated Hospital of Soochow University. Written informed consent was obtained from each participant before inclusion in the study.

\section{Sleep evaluation}

All participants underwent full-night PSG using the Voyager Digital Imaging E-series system (Compumedics, 
Melbourne, Australia). OSA was diagnosed according to an apnea-hypopnea index $(\mathrm{AHI}) \geq 5(21,30)$.

\section{Collection of clinical data}

The age and sex of each participant were recorded. Patients who smoked at least 1 cigarette per day during the study were regarded as smokers. Waist circumference was measured at the level of the umbilicus, hip circumference was measured at the level of maximum extension of the hip, and the waist-to-hip ratio (WHR) was determined from these values. BMI was calculated by dividing weight in kilograms by the square of height in meters. Blood pressure was measured with a mercury sphygmomanometer after the patient had been seated quietly for at least $10 \mathrm{~min}$. A participant was considered to have hypertension if their blood pressure was $>140 / 90 \mathrm{mmHg}$ or if they were taking any anti-hypertensive drugs. Venous blood samples were obtained in the morning after an overnight fast, and standard laboratory procedures were used to measure the following biochemical parameters: alanine transaminase (ALT), aspartate transaminase (AST), gamma-glutamyl transferase (GGT), lactate dehydrogenase (LDH), blood urea nitrogen (BUN), serum creatinine (CR), fasting C-peptide (FCP), fasting plasma glucose (FPG), total cholesterol (TC), high-density lipoprotein (HDL), lowdensity lipoprotein (LDL), triglycerides (TG), and uric acid (UA). The presence/absence of fatty liver was determined based on the medical history and ultrasonography. The homeostasis model assessment 2 insulin resistance index (HOMA2-IR) was calculated using software downloaded from http://www.dtu.ox.ac.uk.

\section{Statistical analysis}

The analysis was conducted using R 3.6.1.3 (TUNA Team, Tsinghua University, China), SPSS 24.0 (IBM, Armonk, NY, USA) and SAS 9.2 (SAS Institute, Cary, NC, USA). A random number table generated by SAS 9.2 was used to randomly assign $70 \%$ of the participants to the training cohort and $30 \%$ of the participants to the validation cohort. The logistic regression model was used to estimate the hazard ratio and corresponding [95\% confidence interval (CI)] for risk factors. Selection methods (forward and exclusion criteria of type I error $=0.1$ based on likelihood ratio tests) were considered in the multivariate model to build the risk prediction model. $\mathrm{P}$ values were two-sided, and values of $<0.05$ were considered statistically significant. We used the glmnet package in $\mathrm{R}$ to perform least absolute shrinkage and selection operator (LASSO) logistic regression, which is suitable for highdimensional, low-sample size data with collinearity issues. Significant factors identified by LASSO regression were used to construct a nomogram to identify patients at risk of OSA (31). Validation was carried out using 1,000 bootstrap replications. Calibration diagrams were assessed as described in a previous report (32). The predictive and discriminatory ability of this model were evaluated by calculation of an index measuring the probability of concordance between the predicted and actual outcomes (C-index) (33). Model calibration was assessed based on Copas's proposal (34) of using a regression smoothing method to produce calibration plots that graphically describe the relationship between the observed and predicted probabilities of OSA. A two-sided P value $<0.05$ was considered statistically significant.

\section{Results}

\section{Clinical characteristics of the study participants}

Among the 414 patients screened for inclusion, 16 patients were excluded because they did not undergo PSG. Therefore, a total of 398 patients (279 men, $70.1 \%$ ) were included in the final analysis. The clinical characteristics of the study participants are summarized in Table 1. The overall prevalence of OSA was $58.5 \%$. Among the patients with OSA, the sleep disorder was considered to be severe in $45.5 \%$, moderate in $29.6 \%$, and mild in $24.9 \%$. A total of 280 participants $(70 \%)$ were randomly assigned to the training cohort, and 118 participants (30\%) were assigned to the validation cohort. There were no significant differences between the training and validation cohorts in demographic and clinical characteristics. However, when compared with patients without OSA, patients with OSA had a higher proportion of males; a higher proportion of smokers; a greater prevalence of fatty liver; a higher BMI; a larger hip circumference; a larger waist circumference; a greater WHR; higher levels of AST, LDH, FCP, TG, and UA; a lower level of HDL; and a greater HOMA2-IR $(\mathrm{P}<0.05$ for all parameters in both the training and validation cohorts; Table 1). Additional significant differences between patients with OSA and those without OSA were observed for prevalence of hypertension (validation cohort), TC (validation cohort), and AST (training cohort). In the training group, univariate analysis and multivariate analysis were performed to identify the risk factors associated with OSA in type 2 diabetes patients (Table 2). 
Table 1 Clinical characteristics of the study participants

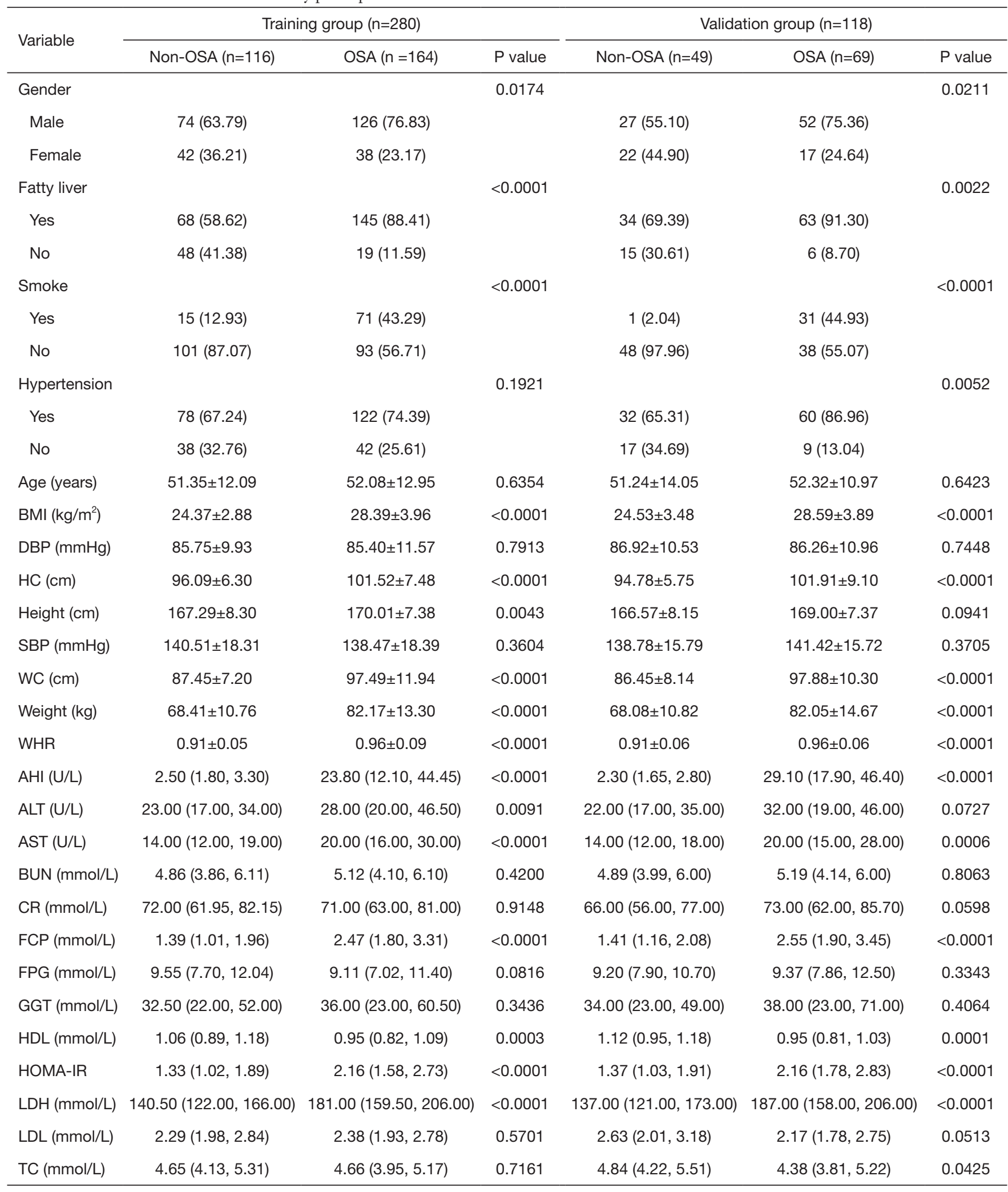

Table 1 (continued) 
Table 1 (continued)

\begin{tabular}{|c|c|c|c|c|c|c|}
\hline Variable & \multicolumn{3}{|c|}{ Training group $(n=280)$} & \multicolumn{3}{|c|}{ Validation group $(n=118)$} \\
\hline $\mathrm{TG}(\mathrm{mmol} / \mathrm{L})$ & $1.90(1.44,3.06)$ & $2.46(1.82,3.97)$ & 0.0003 & $2.08(1.45,2.86)$ & $2.71(1.82,3.90)$ & 0.0083 \\
\hline UA (mmol/L) & $242.55(202.75,296.55)$ & $326.00(267.65,393.15)$ & $<0.0001$ & $235.10(198.30,285.80)$ & $335.80(274.70,395.90)$ & $<0.0001$ \\
\hline
\end{tabular}

Data are presented as mean \pm standard deviation, median (interquartile range), or number (percentage). Baseline characteristics were compared between the two groups using the t-test for independent samples (normally distributed continuous variables), Mann-Whitney $\mathrm{U}$ test (non-normally distributed continuous variables), or chi-squared test or Fisher's exact test (categorical variables). OSA, obstructive sleep apnea; AHI, apnea-hypopnea index; ALT, alanine transaminase; AST, aspartate transaminase; BMI, body mass index; CR, creatinine; DBP, diastolic blood pressure; FCP, fasting C-peptide; FPG, fasting plasma glucose; GGT, gamma-glutamyl transferase; HC, hip circumference; HDL, high-density lipoprotein cholesterol; HOMA-IR, homeostasis model assessment of insulin resistance; LDH lactate dehydrogenase; LDL low-density lipoprotein cholesterol; SBP, systolic blood pressure; TC, cholesterol; TG, triglycerides; UA, uric acid; WC, waist circumference; WHR, waist-to-hip ratio.

Table 2 Risk factors for OSA in type 2 diabetic patients according to the logistic regression model

\begin{tabular}{|c|c|c|c|c|c|c|}
\hline Variable & \multicolumn{3}{|c|}{ Univariate analysis } & \multicolumn{3}{|c|}{ Multivariate analysis } \\
\hline WHR & $518,994.834$ & $3,891.605-69,214,534.6$ & $<0.001$ & $22,435.245$ & $19.925-25,224,295.12$ & 0.005 \\
\hline Smoke & 5.141 & $2.754-9.595$ & $<0.001$ & 1.812 & $0.8-4.102$ & 0.154 \\
\hline Gender & 0.531 & $0.315-0.898$ & 0.018 & 0.699 & $0.316-1.545$ & 0.376 \\
\hline Hypertension & 1.415 & $0.839-2.387$ & 0.193 & NA & & \\
\hline BMI & 1.453 & $1.315-1.606$ & $<0.001$ & 1.29 & $1.142-1.458$ & $<0.001$ \\
\hline AST & 1.083 & $1.048-1.118$ & $<0.001$ & 1.147 & $1.06-1.242$ & $<0.001$ \\
\hline GGT & 1.002 & $0.997-1.008$ & 0.397 & NA & & \\
\hline HDL & 0.172 & $0.056-0.53$ & 0.002 & 0.908 & $0.318-2.593$ & 0.857 \\
\hline LDL & 0.906 & $0.629-1.305$ & 0.596 & NA & & \\
\hline CR & 1.003 & $0.989-1.017$ & 0.665 & NA & & \\
\hline UA & 1.015 & $1.011-1.019$ & $<0.001$ & 1.008 & $1.002-1.014$ & 0.00497 \\
\hline HOMA-IR & 2.279 & $1.643-3.16$ & $<0.001$ & 1.300 & $0.886-1.907$ & 0.17972 \\
\hline $\mathrm{HbA1c}$ & 0.825 & $0.742-0.918$ & $<0.001$ & 0.966 & $0.88-1.061$ & 0.47126 \\
\hline Fatter liver & 5.387 & $2.944-9.858$ & $<0.001$ & 2.321 & $0.967-5.57$ & 0.05953 \\
\hline
\end{tabular}

OSA, obstructive sleep apnea; NA, not available; WHR, waist-to-hip ratio; BMI, body mass index; AST, aspartate transaminase; GGT, gamma-glutamyl transferase; TC, cholesterol; TG, triglycerides; HDL, high-density lipoprotein cholesterol; LDL low-density lipoprotein cholesterol; CR, creatinine; UA, uric acid; HOMA-IR, homeostasis model assessment of insulin resistance.

\section{Factor selection for the predictive model}

The LASSO method is a shrinkage estimation method that can be used to select relevant features in high-dimensional data. Compression coefficients are obtained by constructing a penalty function, and some of the compression coefficients are set to zero to establish a more refined linear regression 

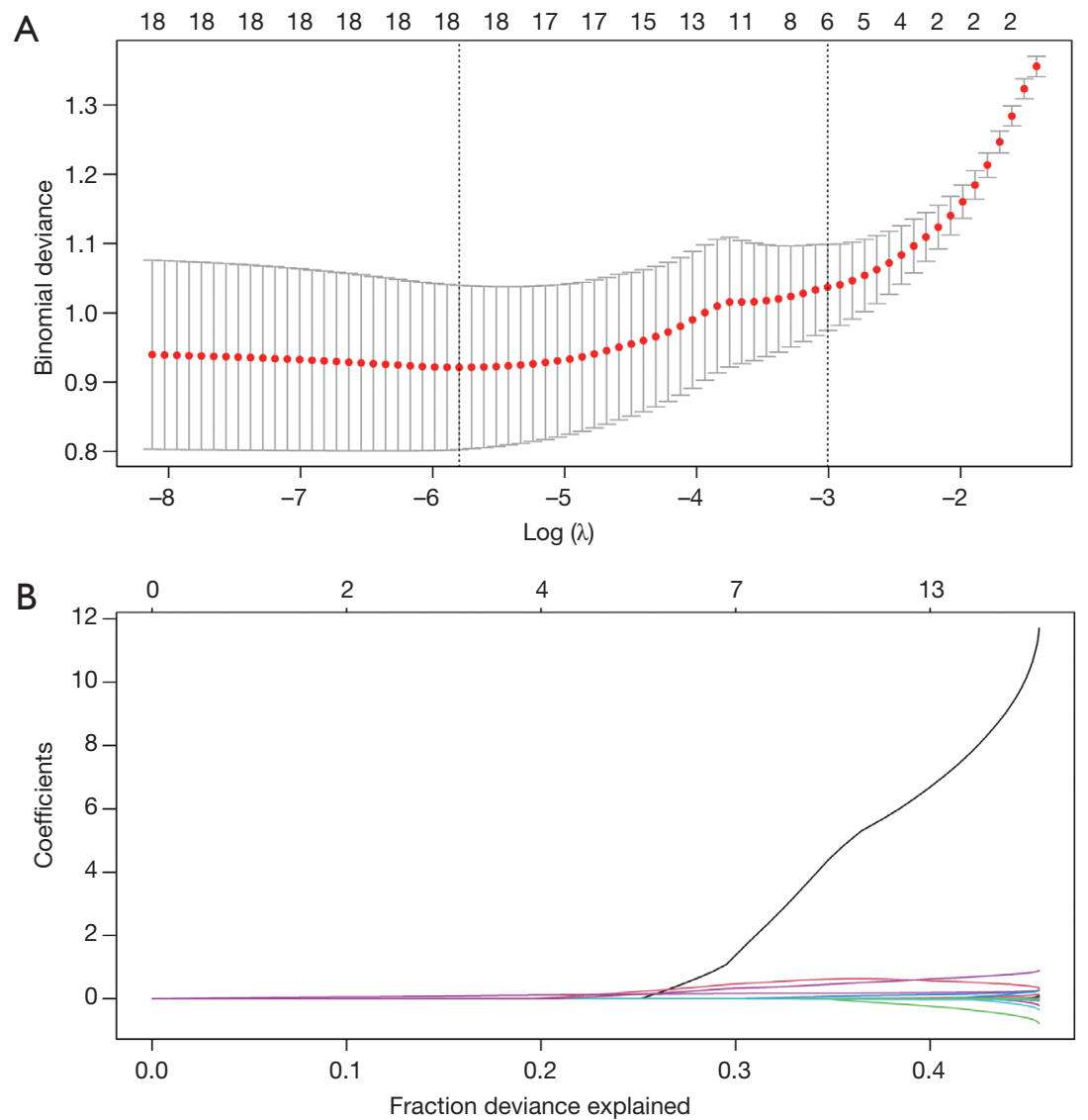

Figure 1 LASSO feature selection model. (A) LASSO coefficients of 17 candidate variables. (B) Identification of the optimal penalization coefficient $(\lambda)$ in the LASSO model was achieved by 10-fold cross-validation and the minimum criterion. The left vertical line represents the minimum error, and the right vertical line represents the cross-validated error within 1 standard error of the minimum. LASSO, least absolute shrinkage and selection operator.

model. In this study, a risk score was calculated from a linear combination of factors weighted by their coefficients, and a coefficient profile plot was constructed (Figure 1A). A cross-validated error plot of the LASSO regression model is shown in Figure 1B. The most regularized and parsimonious model, with a cross-validated error within 1 standard error of the minimum, included 6 of the 17 variables which were significantly different between two groups. Therefore, a model incorporating 6 independent predictors (WHR, smoking status, BMI, UA, HOMA2-IR, and history of fatty liver) was developed into a simple-to-use nomogram (Figure 2).

\section{Validation of the nomogram}

A 1,000 bootstrap analysis was used to validate the nomogram. The C-index for predicting OSA in patients with T2DM was 0.88 in the training group and 0.88 in the validation group. Since both $\mathrm{C}$-index values exceeded 0.7 , the model was deemed suitable and sufficiently accurate for predicting OSA in patients with T2DM. The calibration plots revealed an excellent correlation between observed and predicted OSA in both the training group (Figure $3 A$ ) and validation group (Figure 3B).

\section{LASSO feature regression model}

A LASSO feature regression model was established to directly visualize the differences between OSA and nonOSA (Figure 4). According to the nomogram, the cutoff point for distinguishing between OSA and non-OSA was 94.43. Therefore, the nomogram score for each participant was standardized using the following formula: standardized 


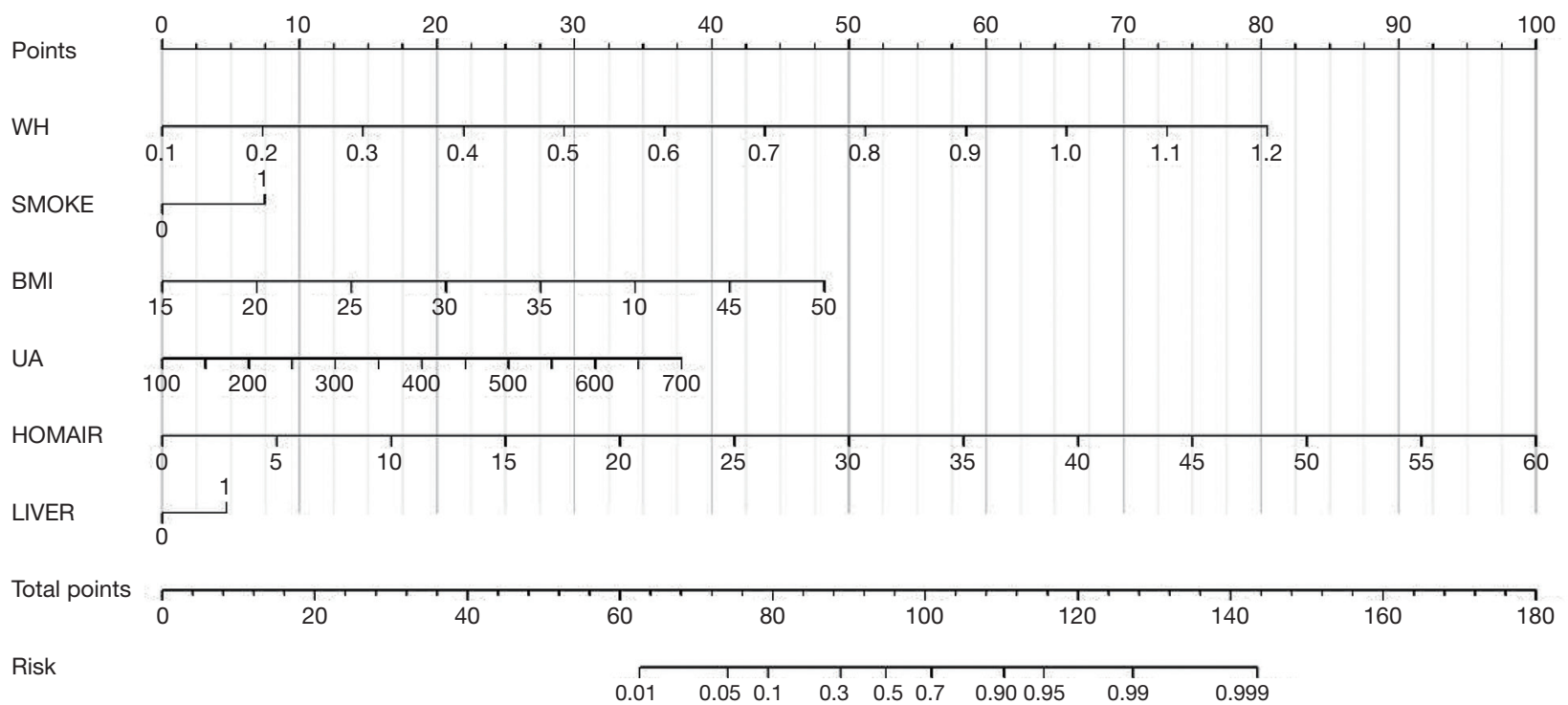

Figure 2 Nomogram constructed for predicting OSA in patients with type 2 diabetes mellitus. OSA, obstructive sleep apnea; BMI, body mass index; HOMA-IR, homeostasis model assessment 2 insulin resistance index; LIVER, history of fatty liver; SMOKE, smoking status; UA, uric acid; WHR, waist-to-hip ratio.
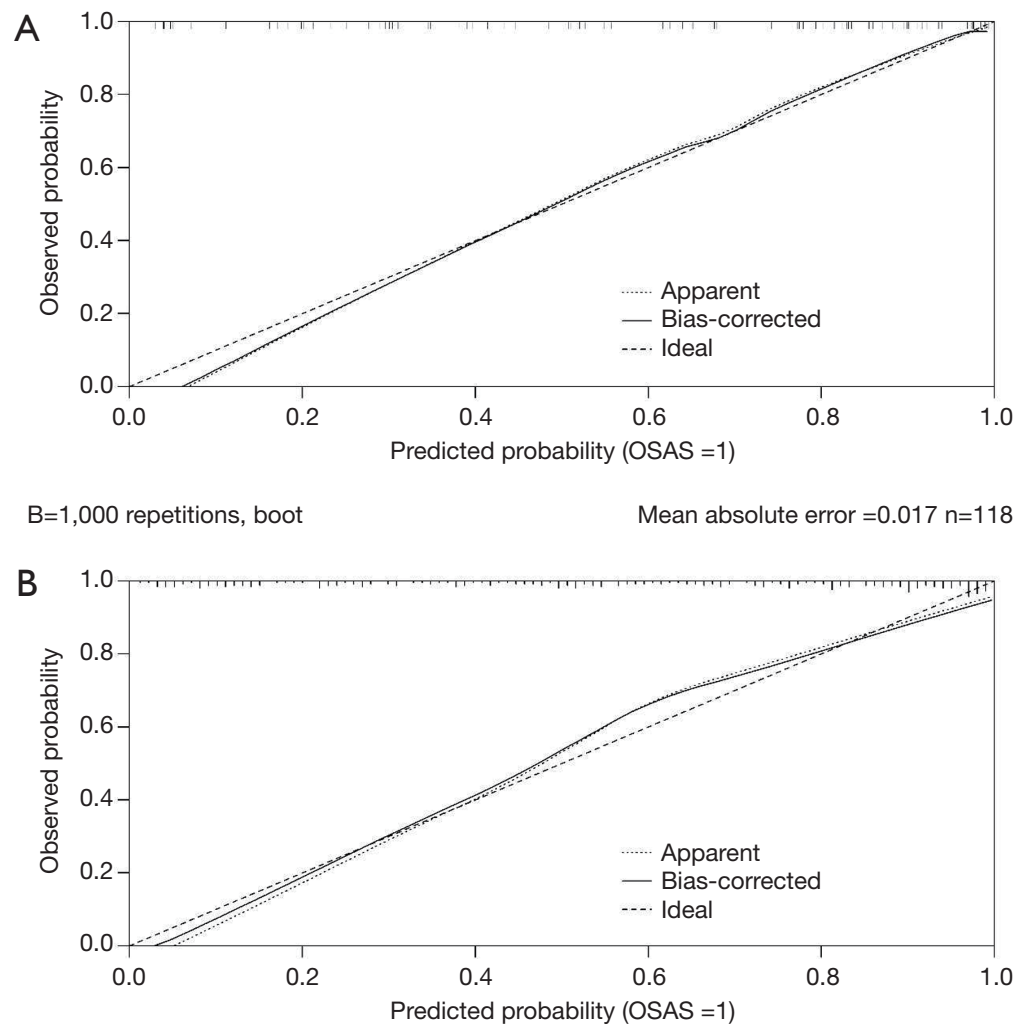

$B=1,000$ repetitions, boot

Mean absolute error $=0.027 \mathrm{n}=288$

Figure 3 Calibration curves for the nomogram. (A) Calibration curve for the nomogram in the training group. (B) Calibration curve for the nomogram in the validation group. 


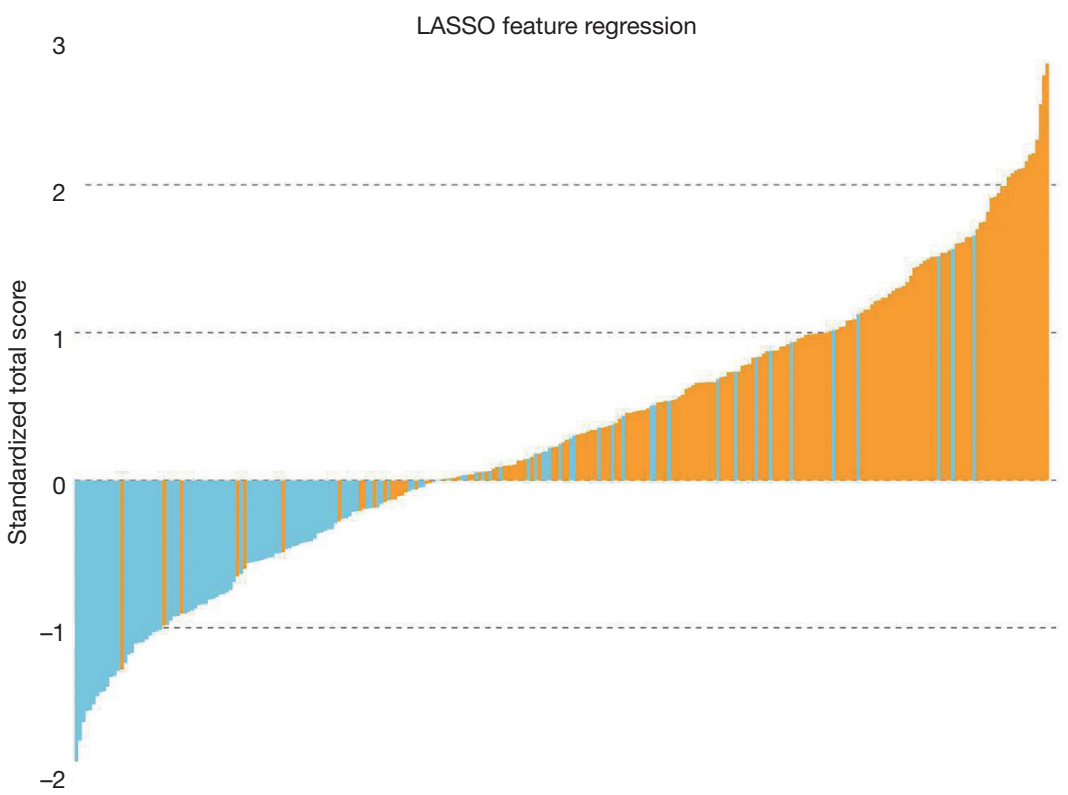

Figure 4 LASSO feature regression. Standardized total score for each participant in the training group. The y-axis represents the calculated value, and the $\mathrm{x}$-axis represents each patient. Green bars represent scores for patients with type 2 diabetes mellitus who do not have obstructive sleep apnea, and red bars represent scores for patients with type 2 diabetes mellitus who do have obstructive sleep apnea. LASSO, least absolute shrinkage and selection operator.

Table 3 The clinical utility of the nomogram in the detection of obstructive sleep apnea

\begin{tabular}{lccccccc}
\hline $\begin{array}{l}\text { Apnea- } \\
\text { hypopnea } \\
\text { index }\end{array}$ & $\begin{array}{c}\text { Area under the } \\
\text { curve }\end{array}$ & Sensitivity & Specific & $\begin{array}{c}\text { Positive } \\
\text { likelihood } \\
\text { ratio }\end{array}$ & $\begin{array}{c}\text { Negative } \\
\text { likelihood ratio }\end{array}$ & $\begin{array}{c}\text { Positive } \\
\text { predictive value }\end{array}$ & $\begin{array}{c}\text { Negative } \\
\text { predictive value }\end{array}$ \\
\hline $15>\mathrm{AHI} \geq 5$ & $0.855(0.794-0.904)$ & $83.93(71.7-92.4)$ & $78.45(69.9-85.5)$ & $3.89(2.7-5.6)$ & $0.20(0.1-0.4)$ & $65.3(53.1-76.1)$ & $91.0(83.6-95.8)$ \\
$30>\mathrm{AHI} \geq 15$ & $0.874(0.810-0.922)$ & $88.57(73.3-96.8)$ & $75.00(66.1-82.6)$ & $3.54(2.5-5.0)$ & $0.15(0.06-0.4)$ & $51.7(38.4-64.8)$ & $95.6(89.1-98.8)$ \\
$>30$ & $0.905(0.854-0.943)$ & $86.30(76.2-93.2)$ & $80.17(71.7-87.0)$ & $4.35(3.0-6.3)$ & $0.17(0.10-0.3)$ & $73.3(62.6-82.2)$ & $90.3(82.9-95.2)$ \\
\hline
\end{tabular}

score $=($ nomogram score -94.43$) /$ standard deviation . Since our study included more participants with OSA than participants without OSA, this resulted in the accumulation of colored blocks in the figure. Therefore, we randomly selected 164 patients with both T2DM and OSA and 116 patients with T2DM but without OSA to establish the LASSO feature regression model (Figure 4).

\section{Clinical utility of the nomogram in the prediction of OSA, moderate-to-severe OSA, and severe OSA}

Receiver operating characteristic (ROC) curve analysis was used to evaluate the utility of the nomogram in the prediction of modest OSA $(15>\mathrm{AHI} \geq 5)$, moderate-to- severe OSA $(30>\mathrm{AHI} \geq 15)$ and severe OSA (AHI $\geq 30)$ in patients with T2DM. The area under the curve (AUC) values at the optimal diagnostic cutoff points were 0.851 for predicting OSA, 0.868 for predicting moderate-tosevere OSA, and 0.907 for predicting severe OSA (Table 3), indicating that the nomogram performed well.

\section{An example of nomogram usage}

To illustrate the use of the nomogram, here we provide an example of a patient with the following clinical characteristics: active smoker, history of fatty liver, waist circumference $=96 \mathrm{~cm}$, hip circumference $=100 \mathrm{~cm}, W H R$ $=0.96$, height $=172 \mathrm{~cm}$, weight $=80 \mathrm{~kg}, \mathrm{BMI}=27.04 \mathrm{~kg} / \mathrm{m}^{2}, \mathrm{UA}$ 
$=240.7 \mu \mathrm{mol} / \mathrm{L}, \mathrm{FPG}=4.37 \mathrm{mmol} / \mathrm{L}, \mathrm{FCP}=531.6 \mathrm{pmol} / \mathrm{L}$, and HOMA2-IR $=1.12$. By applying the above values to the nomogram, a total score of 102.37 was obtained, which corresponded to a $72 \%$ probability of having OSA.

\section{Discussion}

To our knowledge, this is the first study to develop an easyto-use nomogram that predicts the risk of OSA in patients with T2DM. The nomogram developed using data from the training group incorporates 6 items, namely WHR, smoking status, BMI, UA, HOMA2-IR, and history of fatty liver. Furthermore, validation demonstrated that the nomogram has good accuracy and discriminatory ability. Our novel nomogram could be used at the bedside or in an outpatient setting to perform a rapid assessment of OSA risk and help identify patients that would normally be referred for diagnostic PSG, which is a more costly and time-consuming examination. The widespread use of this nomogram in patients with T2DM could potentially avoid unnecessary PSG examinations, thereby reducing the burden on patients and healthcare services.

The 17 candidate variables initially used for construction of the nomogram were reduced to 6 potential predictors using the LASSO regression method. The LASSO regression technique is suitable for analyzing large sets of clinical factors and avoids overfitting (35). Our nomogram suggests that smoking, obesity, and disordered glucose metabolism may be good predictors of OSA in patients with T2DM. Tobacco smoking may increase the severity of OSA through alterations in sleep architecture, upper airway neuromuscular function, and arousal mechanisms, as well as induction of upper airway inflammation (36). Although smoking cessation might be expected to improve OSA, the evidence to support this is limited (37); hence, exsmokers may still be at higher risk of OSA. Nonalcoholic fatty liver disease (NAFLD) is closely associated with metabolic syndrome, which appears to be a driving force for a multitude of comorbidities such as insulin resistance and OSA (38). OSA is highly prevalent in patients with metabolic syndrome (39), and an increasing severity of OSA is associated with poorer control of T2DM, hypertension, and dyslipidemia, all of which are components of metabolic syndrome (40). Obesity is considered an important risk factor for OSA, and several large-scale studies have concluded that an increase in BMI is associated with an increasing prevalence and severity of OSA (41-43). Medical treatments and bariatric surgery to achieve weight loss have been shown to alleviate OSA severity, thereby confirming a cause-effect relationship between these two disorders (44). Furthermore, insulin resistance is a key factor in the pathogenesis of T2DM and other OSA-associated metabolic perturbations. In patients with OSA and T2DM, the effects of sleep-disordered breathing on glucose metabolism and the underlying mechanisms have yet to be thoroughly investigated. Nevertheless, in agreement with the findings of our study, previous research has demonstrated that severe OSA is associated with higher HOMA-IR (45).

Several questionnaires have been developed with the aim of identifying patients at high risk of OSA who should be prioritized for PSG, such as the Berlin questionnaire, the Rome questionnaire, and the BASH'IM score, but these tools appear to have inadequate sensitivity or specificity $(23,46,47)$. Three-dimensional cone-beam computerized tomographic airway analysis has also been evaluated as a tool to study the presence and severity of OSA, but this is not widely used because of its high cost (48). Kolotkin et al. constructed an alternative prediction model in patients with OSA undergoing bariatric surgery, but the sensitivity and specificity of this model were not high in the validation group (49). Magalang et al. utilized overnight pulse oximetry to improve the precision of a prediction model, but this method was time-consuming and labor intensive (50). Improvements in statistical modeling have resulted in the development of nomograms to assess OSA risk based on clinical syndromes, demographic characteristics, and anthropometric measures, and these approaches have been demonstrated to have reasonably good accuracy. Luo et al. utilized an ordinal logistic regression procedure to establish a nomogram encompassing numerous subjective variables and found that the discriminatory accuracy of this nomogram for non-OSA, moderate-severe OSA, and severe OSA was $83.8 \%, 79.9 \%$, and $80.5 \%$, respectively (25). $\mathrm{Xu}$ et al. used a LASSO regression technique to construct a nomogram that incorporated 8 independent factors (age, sex, glucose, apolipoprotein B, insulin, BMI, neck circumference, and waist circumference) and predicted nonOSA, moderate-to-severe OSA, and severe OSA with an accuracy of $77 \%, 80 \%$, and $79 \%$, respectively (26).

Whereas the populations enrolled in previous studies comprised participants suspected of having OSA, the present study specifically recruited patients with T2DM because this population is known to have a high prevalence of OSA. Since endocrinologists encounter a large number of patients with T2DM, there is a window of opportunity to screen patients with T2DM on endocrinology wards 
for various comorbidities, including OSA, and implement appropriate interventions as necessary. The nomogram developed in this study performed well as a screening tool to identify patients with T2DM at risk of OSA, as demonstrated by AUC values $>0.8$. The 6 -factor nomogram was established using a LASSO model to reduce overfitting, and calibration plots demonstrated excellent correlation between the predicted and observed probabilities of OSA. We believe that this nomogram could be an effective screening tool to identify patients with T2DM who are at high risk of OSA and thus should be referred for PSG.

This study has some limitations that should be taken into account when interpreting the data. First, the training group and validation group were recruited from the same single center, so whether or not these findings can be generalized to other populations is not clear. It will be important to validate our nomogram at other institutions in China and abroad before it can be widely implemented in clinical practice. Second, the construction of our nomogram was based only on demographic, anthropometric, and biochemical parameters, and other variables such as genetic factors were not considered. Third, the high prevalence of OSA in our study population may have affected the evaluation of the predictive parameters. Fourth, the inclusion of biochemical parameters makes the use of this nomogram somewhat more complex and time-consuming than simple questionnaires. Nevertheless, since routine measurement of these biochemical parameters is far more straightforward than PSG, improving the predictive accuracy of the nomogram by including these biochemical parameters likely outweighs the disadvantage of performing these blood tests.

The present study has established a novel nomogram to predict the risk of OSA in patients with T2DM admitted to an endocrinology ward. Although the sensitivity and specificity of this nomogram need further evaluation in the general population, we anticipate that this nomogram could be used as a screening tool to identify patients with T2DM who are at risk for OSA and thus merit referral for PSG.

\section{Acknowledgments}

Funding: This work was supported by the Maternal and Child Health Project of Jiangsu Province (No. F201803), the Six talent peaks project in Jiangsu Province (No. WSW-183), and the Major Project of Changzhou Health Commission (No. ZD201903).

\section{Footnote}

Reporting Checklist: The authors have completed the TRIPOD reporting checklist. Available at http://dx.doi. org/10.21037/atm-20-6890

Data Sharing Statement: Available at http://dx.doi. org/10.21037/atm-20-6890

Conflicts of Interest: All authors have completed the ICMJE uniform disclosure form (available at http://dx.doi. org/10.21037/atm-20-6890). FH served as an editor board member of Annals of Translational Medicine from Mar 2017 to Feb 2019 and associate editor-in-chief of Annals of Translational Medicine from Mar 2020 to Mar 2021. The other authors have no conflicts of interest to declare.

Ethical Statement: The authors are accountable for all aspects of the work in ensuring that questions related to the accuracy or integrity of any part of the work are appropriately investigated and resolved. All procedures performed in this study involving human participants were in accordance with the Declaration of Helsinki (as revised in 2013). The protocol was approved by the Ethics Committee of the Third Affiliated Hospital of Soochow University. Written informed consent was obtained from each participant before inclusion in the study.

Open Access Statement: This is an Open Access article distributed in accordance with the Creative Commons Attribution-NonCommercial-NoDerivs 4.0 International License (CC BY-NC-ND 4.0), which permits the noncommercial replication and distribution of the article with the strict proviso that no changes or edits are made and the original work is properly cited (including links to both the formal publication through the relevant DOI and the license). See: https://creativecommons.org/licenses/by-nc-nd/4.0/.

\section{References}

1. Gaines J, Vgontzas AN, Fernandez-Mendoza J, et al. Obstructive sleep apnea and the metabolic syndrome: The road to clinically-meaningful phenotyping, improved prognosis, and personalized treatment. Sleep Med Rev 2018;42:211-9.

2. Maspero C, Giannini L, Galbiati G, et al. Obstructive sleep apnea syndrome: a literature review. Minerva Stomatol 2015;64:97-109. 
3. Senaratna CV, Perret JL, Lodge CJ, et al. Prevalence of obstructive sleep apnea in the general population: A systematic review. Sleep Med Rev 2017;34:70-81.

4. Xu ZF, Luo X, Shi J, et al. Quality analysis of smart phone sleep apps in China: can apps be used to conveniently screen for obstructive sleep apnea at home? BMC Med Inform Decis Mak 2019;19:224.

5. Liao WJ, Song LJ, Yi HL, et al. Treatment choice by patients with obstructive sleep apnea: data from two centers in China. J Thorac Dis 2018;10:1941-50.

6. Costantino A, Rinaldi V, Moffa A, et al. Hypoglossal nerve stimulation long-term clinical outcomes: a systematic review and meta-analysis. Sleep Breath 2020;24:399-411.

7. Eckert DJ. Phenotypic approaches to obstructive sleep apnoea - New pathways for targeted therapy. Sleep Med Rev 2018;37:45-59.

8. Dudley KA, Patel SR. Disparities and genetic risk factors in obstructive sleep apnea. Sleep Med 2016;18:96-102 .

9. Gottlieb DJ, Punjabi NM. Diagnosis and Management of Obstructive Sleep Apnea: A Review. Jama 2020;323:1389-400.

10. Bakker JP, Weng J, Wang R, et al. Associations between Obstructive Sleep Apnea, Sleep Duration, and Abnormal Fasting Glucose. The Multi-Ethnic Study of Atherosclerosis. Am J Respir Crit Care Med 2015;192:745-53.

11. Pugliese G, Barrea L, Laudisio D, et al. Sleep Apnea, Obesity, and Disturbed Glucose Homeostasis: Epidemiologic Evidence, Biologic Insights, and Therapeutic Strategies. Curr Obes Rep 2020;9:30-8.

12. Leong WB, Jadhakhan F, Taheri S, et al. The Association between Obstructive Sleep Apnea on Diabetic Kidney Disease: A Systematic Review and Meta-Analysis. Sleep 2016;39:301-8.

13. Xu S, Wan Y, Xu M, et al. The association between obstructive sleep apnea and metabolic syndrome: a systematic review and meta-analysis. BMC Pulm Med 2015;15:105.

14. Wang X, Bi Y, Zhang Q, et al. Obstructive sleep apnoea and the risk of type 2 diabetes: a meta-analysis of prospective cohort studies. Respirology 2013;18:140-6.

15. Rajan P, Greenberg H. Obstructive sleep apnea as a risk factor for type 2 diabetes mellitus. Nat Sci Sleep 2015;7:113-25.

16. Greco C, Spallone V. Obstructive Sleep Apnoea Syndrome and Diabetes. Fortuitous Association or Interaction? Curr Diabetes Rev 2015;12:129-55.

17. Veasey SC, Rosen IM. Obstructive Sleep Apnea in Adults.
N Engl J Med 2019;380:1442-9.

18. Zhang R, Guo X, Guo L, et al. Prevalence and associated factors of obstructive sleep apnea in hospitalized patients with type 2 diabetes in Beijing, China 2. J Diabetes 2015;7:16-23.

19. Zhang $\mathrm{P}$, Zhang R, Zhao F, et al. The prevalence and characteristics of obstructive sleep apnea in hospitalized patients with type 2 diabetes in China. J Sleep Res 2016;25:39-46.

20. Xie W, Zheng F, Song X. Obstructive sleep apnea and serious adverse outcomes in patients with cardiovascular or cerebrovascular disease: a PRISMA-compliant systematic review and meta-analysis. Medicine (Baltimore) 2014;93:e336.

21. Goyal M, Johnson J. Obstructive Sleep Apnea Diagnosis and Management. Mo Med 2017;114:120-4.

22. Flemons WW, Douglas NJ, Kuna ST, et al. Access to diagnosis and treatment of patients with suspected sleep apnea. Am J Respir Crit Care Med 2004;169:668-72.

23. Chiu HY, Chen PY, Chuang LP, et al. Diagnostic accuracy of the Berlin questionnaire, STOP-BANG, STOP, and Epworth sleepiness scale in detecting obstructive sleep apnea: A bivariate meta-analysis. Sleep Med Rev 2017;36:57-70.

24. Bibbins-Domingo K, Grossman DC, Curry SJ, et al. Screening for Obstructive Sleep Apnea in Adults: US Preventive Services Task Force Recommendation Statement. Jama 2017;317:407-14.

25. Luo M, Zheng HY, Zhang Y, et al. A Nomogram for Predicting the Likelihood of Obstructive Sleep Apnea to Reduce the Unnecessary Polysomnography Examinations. Chin Med J (Engl) 2015;128:2134-40.

26. Xu H, Zhao X, Shi Y, et al. Development and validation of a simple-to-use clinical nomogram for predicting obstructive sleep apnea. BMC Pulm Med 2019;19:18.

27. Bucca C, Brussino L, Maule MM, et al. Clinical and functional prediction of moderate to severe obstructive sleep apnoea. Clin Respir J 2011;5:219-26.

28. Ahlin S, Manco M, Panunzi S, et al. A new sensitive and accurate model to predict moderate to severe obstructive sleep apnea in patients with obesity. Medicine (Baltimore) 2019;98:e16687.

29. American Diabetes Association. 2. Classification and Diagnosis of Diabetes: Standards of Medical Care in Diabetes-2020. Diabetes Care 2020;43:S14-31.

30. Kapur VK, Auckley DH, Chowdhuri S, et al. Clinical Practice Guideline for Diagnostic Testing for Adult Obstructive Sleep Apnea: An American Academy of Sleep 
Medicine Clinical Practice Guideline. J Clin Sleep Med 2017;13:479-504.

31. Friedman J, Hastie T, Tibshirani R. Regularization Paths for Generalized Linear Models via Coordinate Descent. J Stat Softw 2010;33:1-22.

32. Sauerbrei $W$, Royston $P$, Binder H. Selection of important variables and determination of functional form for continuous predictors in multivariable model building. Stat Med 2007;26:5512-28.

33. Wolbers M, Koller MT, Witteman JC, et al. Prognostic models with competing risks: methods and application to coronary risk prediction. Epidemiology 2009;20:555-61.

34. Copas JB. Regression, Prediction and Shrinkage. Journal of the Royal Statistical Society Series B: Methodological 1983;45:311-35.

35. Hepp T, Schmid M, Gefeller O, et al. Approaches to Regularized Regression - A Comparison between Gradient Boosting and the Lasso. Methods Inf Med 2016;55:422-30.

36. Deleanu OC, Pocora D, Mihălcuţă S, et al. Influence of smoking on sleep and obstructive sleep apnea syndrome. Pneumologia 2016;65:28-35.

37. Zhu HM, Yi HL, Guan J, et al. Relationship between smoking and the severity of OSA. Lin Chung Er Bi Yan Hou Tou Jing Wai Ke Za Zhi 2019;33:862-5;9.

38. Kovalic AJ, Cholankeril G, Satapathy SK. Nonalcoholic fatty liver disease and alcoholic liver disease: metabolic diseases with systemic manifestations. Transl Gastroenterol Hepatol 2019;4:65.

39. Drager LF, Togeiro SM, Polotsky VY, et al. Obstructive sleep apnea: a cardiometabolic risk in obesity and the metabolic syndrome. J Am Coll Cardiol 2013;62:569-76.

40. Soin D, Kumar PA, Chahal J, et al. Evaluation of obstructive sleep apnea in metabolic syndrome. J Family Med Prim Care 2019;8:1580-6.

41. Senaratna CV, English DR, Currier D, et al. Sleep apnoea in Australian men: disease burden, co-morbidities, and correlates from the Australian longitudinal study on male

Cite this article as: Shi H, Xiang S, Huang X, Wang L, Hua F, Jiang X. Development and validation of a nomogram for predicting the risk of obstructive sleep apnea in patients with type 2 diabetes. Ann Transl Med 2020;8(24):1675. doi: 10.21037/atm-20-6890 health. BMC Public Health 2016;16:1029.

42. Peppard PE, Young T, Palta M, et al. Longitudinal study of moderate weight change and sleep-disordered breathing. Jama 2000;284:3015-21.

43. Li C, Ford ES, Zhao G, et al. Prevalence of self-reported clinically diagnosed sleep apnea according to obesity status in men and women: National Health and Nutrition Examination Survey, 2005-2006. Prev Med 2010;51:18-23.

44. Chierakul N, Chaipattarapol C, Ruttanaumpawan P, et al. Comparison of clinical and polysomnographic characteristics of non-obese and obese patients with obstructive sleep apnea. J Med Assoc Thai 2007;90 Suppl 2:48-53.

45. Zhang Y, Xing Y, Yuan H, et al. Impaired Glucose Metabolisms of Patients with Obstructive Sleep Apnea and Type 2 Diabetes. J Diabetes Res 2018;2018:6714392.

46. Ng SS, Tam W, Chan TO, et al. Use of Berlin questionnaire in comparison to polysomnography and home sleep study in patients with obstructive sleep apnea. Respir Res 2019;20:40.

47. Sun LM, Chiu HW, Chuang CY, et al. A prediction model based on an artificial intelligence system for moderate to severe obstructive sleep apnea. Sleep Breath 2011;15:317-23.

48. Enciso R, Nguyen M, Shigeta Y, et al. Comparison of cone-beam CT parameters and sleep questionnaires in sleep apnea patients and control subjects. Oral Surg Oral Med Oral Pathol Oral Radiol Endod 2010;109:285-93.

49. Kolotkin RL, LaMonte MJ, Walker JM, et al. Predicting sleep apnea in bariatric surgery patients. Surg Obes Relat Dis 2011;7:605-10.

50. Magalang UJ, Dmochowski J, Veeramachaneni S, et al. Prediction of the apnea-hypopnea index from overnight pulse oximetry. Chest 2003;124:1694-701.

(English Language Editor: J. Gray) 